\title{
Stacking disorder: the hexagonal polymorph of tris(bicyclo[2.1.1]hexeno)benzene and related examples
}

\author{
Hans-Beat Bürgi*,I ${ }^{*}$ Marc Hostettler ${ }^{\mathrm{I}}$, Henrik Birkedal ${ }^{\mathrm{II}, 1}$ and Dieter Schwarzenbach ${ }^{\mathrm{II}}$ \\ I Laboratorium für Kristallographie der Universität Bern, Freiestrasse 3, 3012 Bern, Switzerland \\ II Laboratoire de Cristallographie, Ecole Polytechnique Fédérale de Lausanne, BSP Dorigny, 1015 Lausanne, Switzerland
}

Received December 24, 2004; accepted April 15, 2005

\section{Tris(bicyclo[2.1.1]hexeno)benzene / Diffuse scattering / Stacking disorder / Polytypism / Polymorphism / $X$-ray diffraction}

\begin{abstract}
X-ray diffractograms of tris(bicyclo[2.1.1]hexeno)benzene, crystallized at the interface between a benzene solution and a layer of acetonitrile, show hexagonal symmetry and streaks of diffuse scattering along $\mathbf{c}^{*}$. The heavily faulted layer stacking is analyzed qualitatively and quantitatively in terms of a systematic protocol. This protocol requires partitioning the crystal structure into layers in such a way that pairs of adjacent layers may be stacked in different, but geometrically equivalent ways, which are dictated by the layer group symmetry. This approach is shown to provide a consistent alternative for analysis of a number of related cases provided the layers are defined on the basis of geometrical criteria rather than chemical intuition.
\end{abstract}

\section{Introduction}

The protocol for elucidating a crystal structure is now so well established that robots are close to replacing crystallographers. Automated procedures for crystallization, collection and quality assessment of diffraction data as well as structure solution and refinement are highly successful and efficiently produce tens of thousands of structures per year - at least for well-ordered crystals. In comparison studies reporting on the microscopic structure of disordered systems are few and far between. The lack of welldefined, user-friendly tools to tackle such problems may well be one of the reasons for this shortcoming.

All real crystals show instantaneous and many show permanent breakdown of translational symmetry resulting in diffuse scattering, which is called 'thermal diffuse scattering' (TDS) and 'static disorder scattering', respectively. Studies of diffuse scattering have largely concentrated on alloys and ionic crystals, many of which show substitu-

\footnotetext{
* Correspondence author (e-mail: hans-beat.buergi@krist.unibe.ch)

1 Present address: Department of Chemistry, University of Århus, 140 Langelandsgade, 8000 Århus C, Denmark
}

tional disorder with different chemical species occupying corresponding sites in different unit cells. In molecular crystals the molecules or parts of them often break translational symmetry by assuming different orientations in different unit cells [1-6].

In this contribution we focus on substances with an especially simple kind of disorder: materials that are built of layers, which are translationally ordered in two dimensions, but stacked in a disordered fashion in the third dimension. Such disorder is often observed when the translational or rotational symmetry of the layers allows several, geometrically equivalent ways of stacking nearestneighbour layers, which may lead to geometrically inequivalent relationships between next-nearest and more distant neighbours. Stacking disorder manifests itself by flocks of parallel diffuse streaks of scattering density along a specific direction of reciprocal space [5]. This kind of disorder is found among inorganic substances and minerals [7-11], molecular crystals [12-18], protein crystals [19-21], e.g. Rubisco [22] and $t$-RNA [23], and even in a virus crystal [24]. This list is not exhaustive; similarly, the discussion in Sect. 3 is limited to examples, which illustrate specific aspects of stacking disorder and which we know well. We apologize for omissions. In Section 2 we analyze the diffuse streaks in the scattering pattern of tris(bicyclo[2.1.1] hexe-no)-benzene, $\mathbf{1}$, in terms of a disordered stacking of 2D periodic layers. In Section 4 we attempt to generalize our method of analysis to a protocol that is also applicable to related problems.

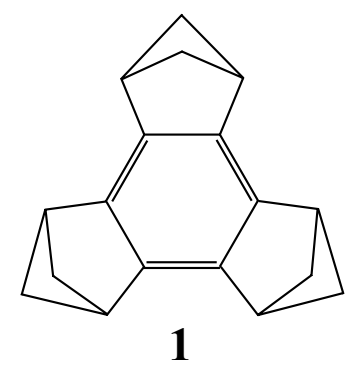

The structure of $\mathbf{1}$ was first determined to study bond alternation in a benzene derivative and was found to be cyclohexatriene-like with a pronounced bond alternation of $\sim 0.09 \AA$. The original work was performed on a sample grown from a benzene solution [25], which showed dis- 
tinct rods of diffuse intensity. Recrystallization of $\mathbf{1}$ at the interface between benzene and acetonitrile produced two different crystal morphologies, a hexagonal one, similar to that grown from benzene, and a new, monoclinic one [26]. The molecular structures from both new crystals were essentially the same as the one found first. Unlike the hexagonal crystals, the monoclinic ones showed nearly no diffuse scattering and an ordered packing of the molecules, but did occur occasionally as growth twins. Ferraris, Makovicky and Merlino have described the stacking faults implied by the presence of the diffuse scattering of $\mathbf{1}$ qualitatively in terms of OD concepts in their book on 'Crystallography of modular materials' [11].

\section{The diffraction pattern and its interpretation}

\subsection{Quantification of the diffraction pattern}

This work is based on the diffraction data described in [26] as $\mathrm{H}$ (hexagonal). The distribution of intensities includes sharp Bragg reflections and strong diffuse streaks

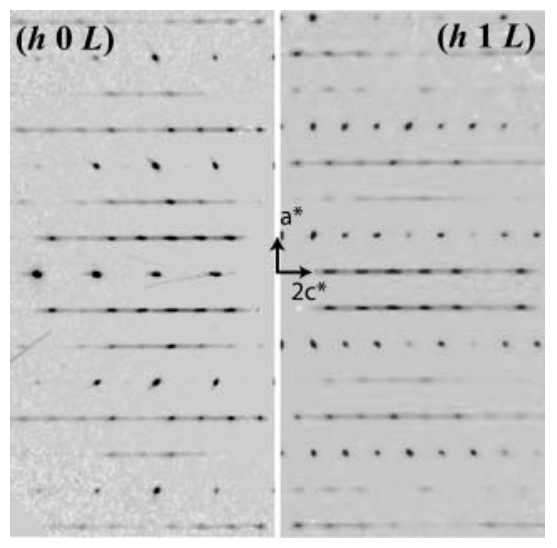

Fig. 1. Layers of reciprocal space for the disordered hexagonal form of 1 reconstructed from image plate data. Indices $h k L$ are assigned relative to a hexagonal coordinate system with $h, k$ integers and $L$ real.

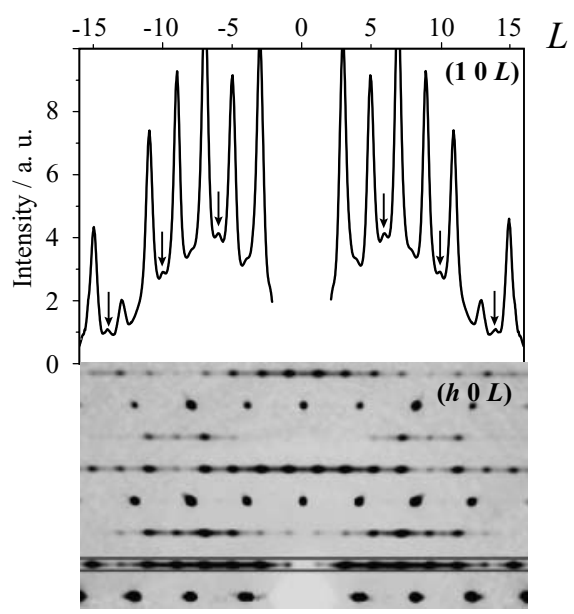

Fig. 2. Extraction of the $(10 L)$ profile of diffuse intensity from the ( $h 0 L$ ) reciprocal layer; at each value of $L$ the intensity is the sum of the intensities from all pixels contained between the two horizontal lines. Note the arrows pointing at some well-resolved small maxima of diffuse intensity observed for $L$ even. as illustrated by the layers of reciprocal space that have been reconstructed with a thickness of one pixel from the raw CCD area detector images using CrysAlis [27]. Fig. 1 shows two such reconstructed layers: $h 0 L$ and $h 1 L$. Profiles of diffuse intensities have been extracted from reciprocal layers using IGOR [28]. Rectangular boxes were defined along the diffuse rods as shown in Fig. 2. At each value of $L$, the intensities inside the box have been integrated in the range $-0.14 \leq h \leq+0.14$. The intensities outside these boxes have been assumed to represent background scattering. The latter was evaluated at each value of $L$ from the average of the two outside profiles integrated in the ranges $-0.42 \leq h \leq-0.14$ and $+0.14 \leq h$ $\leq+0.42$, and subtracted from the intensity profiles. A polarization and an equatorial Lorentz correction have been applied. Note that the reconstruction of layers with one pixel thickness does not guarantee very reliable relative intensities of the strong maxima, but does provide good semi-quantitative data.

\subsection{Interpretation of the diffraction pattern}

The parallel diffuse rods imply that the structure of $\mathbf{1}$ is built from 2D periodic layers, and that the stacking of the layers in the third dimension lacks translational symmetry [5]. The interpretation of such a diffraction pattern is undertaken in two steps. In the first step a so-called average or superposition structure is determined from the Bragg reflections only. Disentanglement of the superposition structure based on crystallographic and chemical arguments provides the structure and symmetry of the 2D periodic layer and the geometric interaction pattern between two consecutive layers expressed in terms of 'local symmetry operations'. Repeated application of the local operations, one at the time, leads to a finite number of periodic crystal structures called structures of maximal degree of order (MDO) [29, 30]. In the second, quantitative step the different stacking sequences are assigned probabilities of occurrence. The probabilities are then optimized to reproduce the observed diffuse rods of intensities. The steps and sub-steps in this procedure are borrowed and adapted from various sources in the literature, primarily from the OD approach of Dornberger-Schiff and from the discussion of interactions between nearest layers, next-nearest layers, etc. as elaborated by many authors over the last several decades $[5,6]$.

\subsubsection{The sharp or Bragg reflections}

The Bragg reflections, also called 'family' reflections, are independent of the stacking sequence of the layers and thus identical for all ordered and disordered structural variants. They show $6 / \mathrm{mmm}$ point symmetry and may be indexed in an hexagonal cell with $a^{\prime}=b^{\prime} \sim 5.2 \AA$ and $c^{\prime}=9.2 \AA$. The latter is called the 'subcell' because it disregards the diffuse part of the diffraction pattern. The systematic absences indicate space group $\mathrm{PG}_{3} / \mathrm{mmc}$ and direct methods reveal two molecules in the subcell centred at $(2 / 3,1 / 3,1 / 4)$ and $(1 / 3,2 / 3,3 / 4)$, and related by an inversion centre at $(1 / 2,1 / 2,1 / 2)$ [25]. Given that the molecular radius of $\mathbf{1}$ is $\sim 4.5 \AA$ neighbouring molecules overlap. Such overlap of 


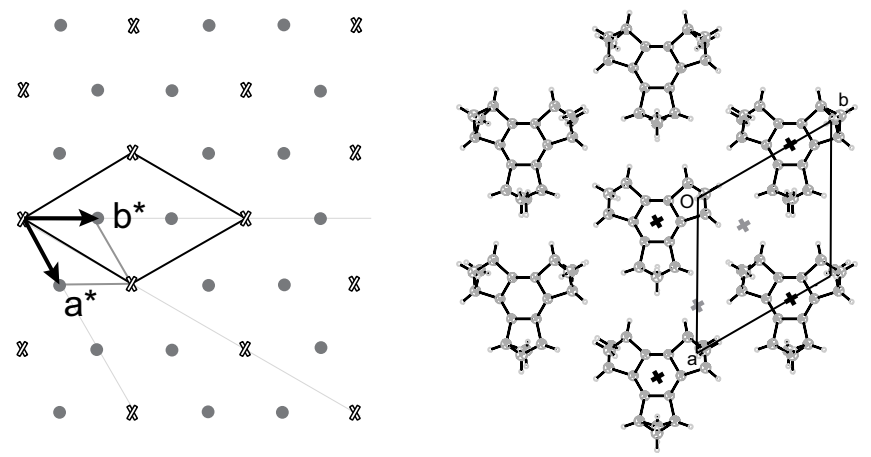

Fig. 3. Left: Schematic drawing of the $h k 0$ layer showing the location of diffuse rods (circles) for $h-k \neq 3$ n and the sharp reflections (crosses) for $h-k=3 n$. The latter define the reciprocal subcell used for the determination of the superposition structure. Right: Average layer structure of 1 with $p(\overline{6}) 2 \mathrm{~m}$ layer symmetry. The centers of the two molecules removed from the superposition structure are depicted as gray crosses.

molecules (and sometimes of atoms) leads to un-chemical 'interatomic distances' thus hampering the interpretation of the electron density distribution obtained from direct methods and making it necessary to use supplementary chemical information to identify the molecular structure which one seeks. The overlap also indicates that the structure determined from the Bragg reflections only represents a particular average over the disordered crystal, the 'superposition structure'.

\subsubsection{Qualitative interpretation of the complete diffraction pattern}

The complete pattern also shows 6/mmm diffraction symmetry. The position of the diffuse streaks relative to the Bragg peaks requires that the unit mesh in the hexagonal plane be $a=b \sim 9.0 \AA$ (Fig. 3). The relation to the subcell is $\mathbf{a}=\mathbf{a}^{\prime}-\mathbf{b}^{\prime}, \mathbf{b}=\mathbf{a}^{\prime}+2 \mathbf{b}^{\prime}$, the reverse relations being $\mathbf{a}^{\prime}=(2 \mathbf{a}+\mathbf{b}) / 3, \mathbf{b}^{\prime}=(-\mathbf{a}+\mathbf{b}) / 3$. Given the diffuse streaks the choice of $\mathbf{c}$ is not unique, but is generally taken as an integer multiple of $\mathbf{c}^{\prime}$; in the present case we choose $\mathbf{c}=2 \mathbf{c}^{\prime}$ which spans the distance of four layers. This choice identifies the broad, but pronounced maxima on the diffuse streaks with (odd) integer values of the continuous reciprocal variable $L$ (Figs. 1 and 2). The diffraction pattern cannot be associated with a space group since the structure lacks translational symmetry.

In the enlarged unit cell the molecular centres of the first layer of the superposition structure are located at $(1 / 3$, $1 / 3,1 / 8),(2 / 3,0,1 / 8),(0,2 / 3,1 / 8)$, and those of the inverted molecules in the second layer at $(2 / 3,2 / 3,3 / 8),(1 / 3,0,3 / 8)$, $(0,1 / 3,3 / 8)$. The tripling of the unit mesh implied in the transformation from $\mathbf{a}^{\prime}, \mathbf{b}^{\prime}$ to $\mathbf{a}, \mathbf{b}$ requires thinning out the occupation of the layers by removing two out of three molecules as shown in Fig. 3 where only the molecule at $(0,2 / 3,1 / 8)$ has been kept. Compared to the superposition structure the distances between the remaining molecular centres are increased by $3^{1 / 2}$, and the intermolecular contacts are now in the range of the usual van der Waals distances. The layer symmetry is $p(\overline{6}) 2 m$, shown schematically in Fig. 4a where the origin has been shifted to the centre of the molecule (note that the symmetry operation

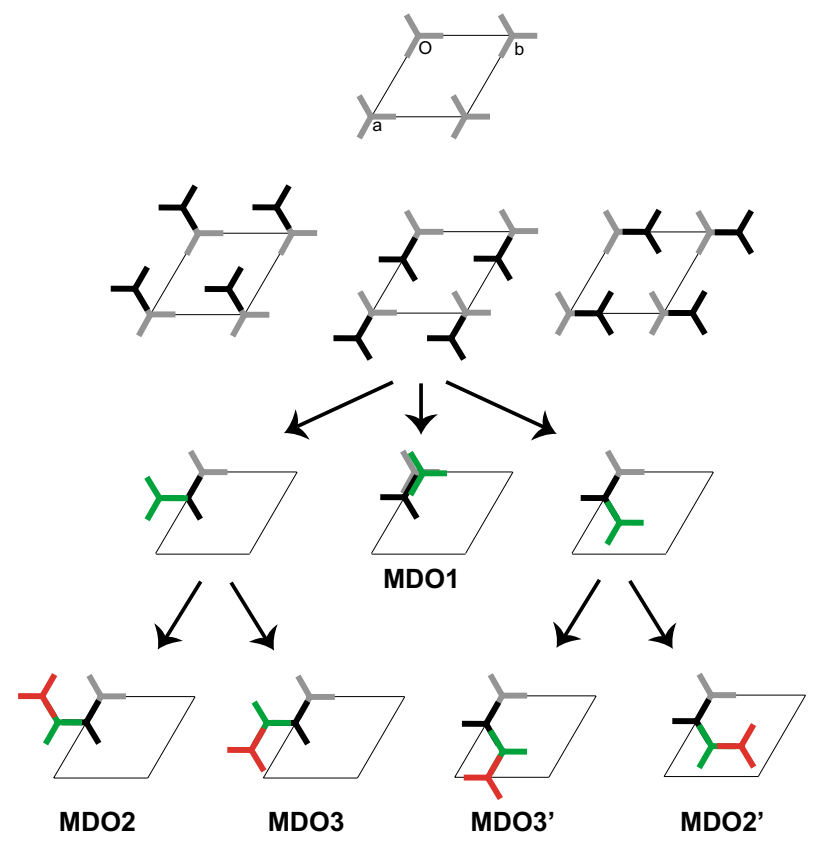

Fig. 4. (a) Schematic representation of a layer of $\mathbf{1}$ with the origin of the unit cell chosen on the $\overline{6}$ axis of the molecule. (b) The three, geometrically equivalent variants to stack a second layer. (c) Stacking the third, green layer eclipsed on top of the first, grey one leads to the unit cell of the orthorhombic structure MDO1, which may again occur in three different orientations (triple twinning). The two alternative ways of stacking the third layer are geometrically equivalent. (d) The two ways of stacking the fourth layer (red) are either characteristic of the hexagonal structures MDO2 with cis stacking (left and right), or of the monoclinic structures MDO3 with trans stacking (centre). For a given position of the second layer the two MDO2 and MDO3 structures are related by the mirror plane $\left(\begin{array}{lll}0 & 1 & 0\end{array}\right)$, implying 2fold twinning for MDO2 and 6-fold twinning for MDO3.

in brackets indicates the direction perpendicular to the layer).

There are three possibilities to choose one molecule in the second layer from the superposition structure (Fig. 4b). The three possibilities are geometrically equivalent under rotation by $120^{\circ}$, implying that there is only one geometrical relationship between nearest neighbour layers. Repeating the selection process in the third layer there are again three possibilities. The first of these puts the molecule in layer three above the molecule in layer one (Fig. 4c, middle), i.e. translated by $\mathbf{c} / 2$. Repeating this stacking pattern we call it eclipsed - leads to a periodic orthorhombic structure with $\mathbf{a}_{\mathrm{o}}=2 \mathbf{a}+\mathbf{b}, \mathbf{b}_{\mathrm{o}}=\mathbf{b}, \mathbf{c}_{\mathrm{o}}=\mathbf{c} / 2$, space group $\mathrm{Cmcm}$ and two layers in the translational unit along $\mathbf{c}$ which are related by a twofold screw operation at $(1 / 6,0$, $z$ ) with translational component $\mathbf{c} / 4$. In the language of OD structures, it is called a structure with maximal degree of order (MDO1). This structure may occur in three different orientations related again by rotations of $120^{\circ}$ and implying the possibility of triple twinning.

The two alternative choices of molecules in layer three lead to the two arrangements in Fig. 4c, left and right we call them bent; they are mutually related by a mirror operation. In both cases there are again two choices for layer four (Fig. 4d). The possibilities on the left and the right may be considered as generated from the repeated application of a 65 operation at $(0,-1 / 3, z)$ with transla- 
tion $-\mathbf{c} / 4$ and $66_{1}$ operation at $(1 / 3,1 / 3, z)$ with translation c/4, respectively. Repeating this stacking pattern - we call it cis - leads to periodic hexagonal structures MDO2 with unit cell $\mathbf{a}_{h}=\mathbf{a}, \mathbf{b}_{h}=\mathbf{b}, \mathbf{c}_{h}=3 \mathbf{c} / 2$. The space groups are $P 6_{5} 22$ or $P 6_{1} 22$ showing that MDO2 may occur as inversion twins. The two possibilities in the middle may be considered as generated by the repeated application of glide operations relative to planes perpendicular to $\mathbf{a}+\mathbf{b}$ or $\mathbf{b}$ and passing through the origin. The glide components are $(1 / 6,-1 / 6,1 / 2)$ and $(1 / 3,1 / 6,1 / 2)$, respectively. When this stacking pattern - we call it trans - is repeated, a monoclinic structure (MDO3) is obtained with space group $C 2 / c$ and unit cell $\mathbf{a}_{m}=\mathbf{a}-\mathbf{b}, \mathbf{b}_{m}=\mathbf{a}+\mathbf{b}$, $\mathbf{c}_{m}=\mathbf{a} / 3-\mathbf{b} / 3+\mathbf{c} / 2$ (or $\mathbf{a}_{m}=2 \mathbf{a}+\mathbf{b}, \mathbf{b}_{m}=\mathbf{b}, \mathbf{c}_{m}=2 \mathbf{a} /$ $3+\mathbf{b} / 3+\mathbf{c} / 2$ ). MDO3 may assume six different orientations related by $120^{\circ}$ rotations and a mirror operation across the [a, c]-plane (Fig. 4d) and thus gives rise to sixfold twinning. We recall that the monoclinic modification shows a nearly ordered MDO3 structure and may occur as growth twin with two orientational domains [26]. There is a fourth type of four-layer sequence consisting of mixed eclipsed and bent arrangements, either an eclipsed followed by a bent arrangement or a bent followed by an eclipsed one. Repeating this type of sequence - we call it faulted - results in an ordered structure which is not an MDO structure. Indeed, this structure has at least two inequivalent layers per asymmetric domain, whereas in an MDO structure all layers are symmetrically equivalent.

The results of this analysis may be summarized as follows: (1) any pair of adjacent layers is geometrically equivalent to any other pair, i.e. there is just one class of pairs of layers; (2) there are two geometrically inequivalent classes of triplets of layers, one of them called eclipsed, the other one bent; and (3) there are four inequivalent classes of quadruplets of layers, one class with two consecutive eclipsed arrangements (repetition gives MDO1), two classes cis and trans deriving from the bent arrangement by successive application of one or the other of two different operations (repetition gives MDO2 or MDO3) and a fourth class - faulted - arising from the use of two different layer-to-layer operations and leading to a more complicated polytype, but not to an MDO-structure. The length $n$ of layer packages to be analysed geometrically depends on the assumed interaction of a given layer with the $(n-1)$-th neighbouring layer and can only be judged on the basis of the probabilistic parameters required for a successful (semi)-quantitative analysis of the diffuse scattering as discussed in the next section.

\subsubsection{Quantitative interpretation of the diffuse streaks}

If the stacking of layers were determined only by the nearest neighbour relationship, all stacking probabilities would assume the same value of $1 / 3$, and the stacking sequence would be random. If next-nearest layers matter, there will be a probability $e$ for the third layer to be eclipsed to the first (MDO1) and $(1-e)$ for it to be bent. If the relative position of the first and fourth layers matters as well, we must introduce two probabilities for $e$, namely $e_{1}$ for stacking eclipsed onto bent and $e_{2}$ for stacking eclipsed onto eclipsed. Thus, $e_{1}$ starts an eclipsed sequence, $e_{2}$ continues an eclipsed sequence, and $1-e_{2}$ starts a bent sequence. We also need probabilities to continue the bent situation in a cis manner $(c)$, and in a trans manner $(t)$. Note the side condition $e_{1}+c+t=1$ and some special cases: $e_{2}=1$ gives MDO1, $c=1 \mathrm{MDO} 2$ and $t=1 \mathrm{MDO} 3$. As will be seen below, these probabilities are necessary and sufficient to interpret quantitatively the observed intensities along the diffuse streaks of $\mathbf{1}$.

This model is an adaptation to an interaction depth of three layers of a simpler model given by Jagodzinski $[31,32]$. It closely resembles the one used for the interpretation of the diffraction data of orange mercuric diiodide $[33,34]$. The intensity distribution along $L$ becomes

$$
\begin{aligned}
I(L)= & 2 M|F|^{2} S(L), \\
S(L)= & (T+U \cos \pi L+V \cos 2 \pi L) /(W+X \cos \pi L \\
& +Y \cos 2 \pi L+Z \cos 3 \pi L) .
\end{aligned}
$$

$F$ is the Fourier transform of a single $p(\overline{6}) 2 m$ layer of molecules of $\mathbf{1}$ in the unit mesh $\mathbf{a}, \mathbf{b}$ with thickness $\mathbf{c}$ corresponding to four interlayer distances. It has been calculated from the coordinates and anisotropic displacement parameters ( $\mathrm{adp}^{\prime} \mathrm{s}$ ) given in Tables 3 and 4 of [26]. $S(L)$ is the interference function with coefficients $T, U, V, W, X, Y$, and $Z$ depending in a complicated way on the probabilities $e_{1}$, $e_{2}, c$ and $t ; M$ is the number of layer pairs (for details see the Appendix). The probabilities and a scale factor have been adjusted by trial and error until the calculated diffuse profiles represented an 'optimal visual fit' to six experimental profiles $I(L)$ as shown in Fig. 5. The probabilities are:
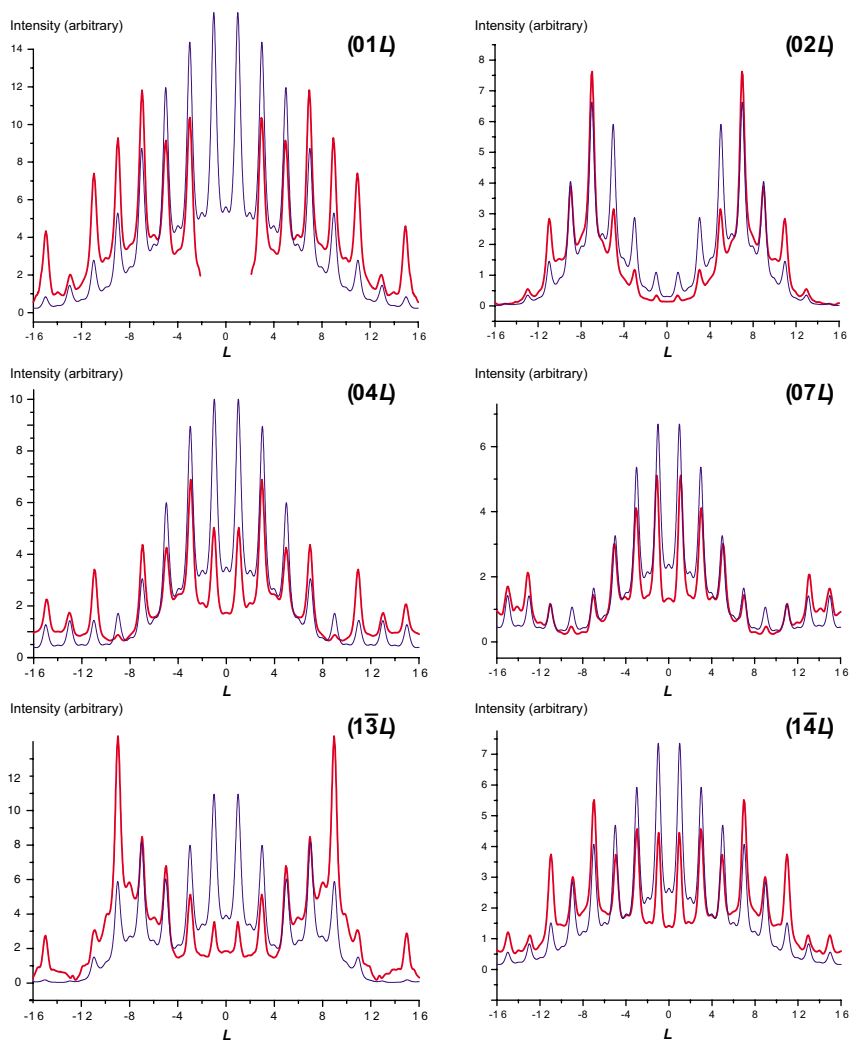

Fig. 5. Comparison of observed profiles (red) with those calculated (blue) from the probabilities $t=0.46, c=0.46, e_{1}=0.08, e_{2}=0.56$. Note that the intensity scale changes from profile to profile. 


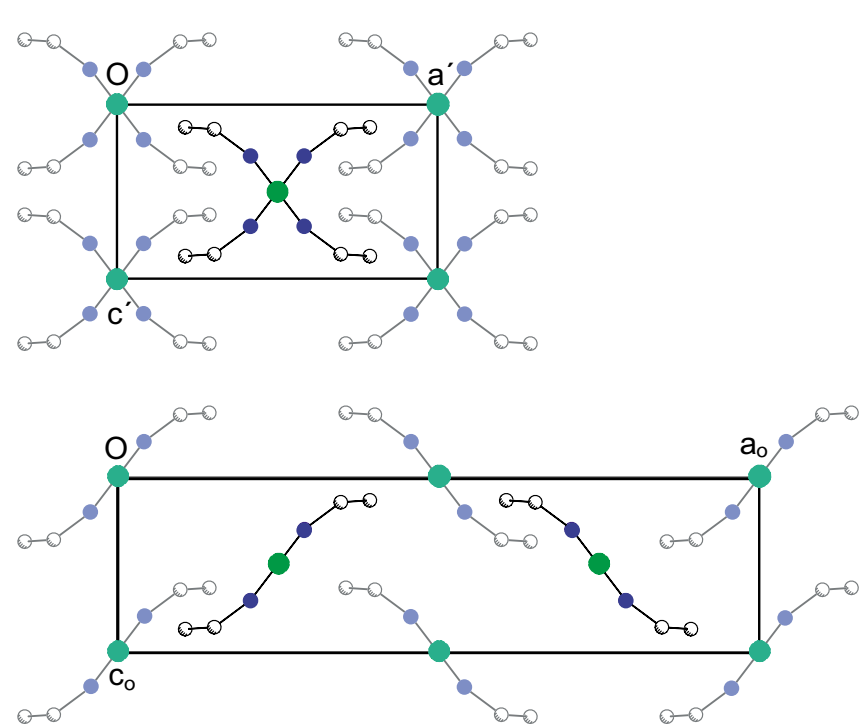

Fig. 6. Top: Schematic drawing of the superposition structure of bis(malondiamidine)nickel(II) dichloride [35] with two molecules superimposed with their $\mathrm{Ni}$-atoms and at approximately right angles. Light molecules at $y^{\prime}=0$; heavy molecules at $y^{\prime}=1 / 2$ (to avoid misleading overlap in the projections the exocyclic amidine nitrogen and the chlorine atoms are not shown). Bottom: Disentanglement to the double layer structure. The heavy molecules are obtained from the light ones either by a 21 screw operation at $(1 / 8, y, 1 / 4)$ or by the translation $\mathbf{a} / 4+\mathbf{b} / 2+\mathbf{c} / 2$. The symmetry of a single layer is $p 2_{1} / m$ (2/m) $2 / a$.

$e_{1}=0.08, e_{2}=0.56, c=t=0.46$. Rough estimates of the reliability of the numerical values of the probabilities are $0.02,0.06$ and 0.1 for $e_{1}, e_{2}$ and $c(t)$, respectively. They have been derived from visual comparisons of $S(L)$ calculated for different probabilities in online version: Fig. Sup. 1-3. The dependence on the probabilities of the shape of the interference function without the influence of the layer form factor is illustrated in the Supplementary Material (Fig. Sup. 4). In Section 4.1 these numbers will be interpreted in terms of preferred stacking sequences, i.e. the main structural features occurring in the sample investigated.

\section{Other examples}

\subsection{Bis(malondiamidine)nickel(II) dichloride}

This square-planar coordination complex, $\left[\mathrm{Ni}\left(\mathrm{C}_{3} \mathrm{H}_{8} \mathrm{~N}_{4}\right)_{2}\right] \mathrm{Cl}_{2}$, shows sharp and diffuse reflections [35]. The former may be indexed in an orthorhombic subcell with $a^{\prime}=9.40$, $b^{\prime}=13.40$ and $c^{\prime}=5.121 \AA$, the latter sit on faint diffuse streaks running parallel to $\mathbf{b}^{\prime *}$ and require a unit mesh of $\mathbf{a}=\mathbf{2} \mathbf{a}^{\prime}, \mathbf{c}=\mathbf{c}^{\prime}$. The superposition structure (Fig. 6, top) shows layers with symmetry $p 2 / m(2 / m) 2 / m$. As for $\mathbf{1}$, there is only one way to disentangle these layers (Fig. 6, bottom). MDO1 with space group $P 2_{1} / n 2_{1} / m 2 / a$ is obtained by repeating the 21 screw operation at $(1 / 8, y, 1 / 4)$ in Fig. 6 (bottom), MDO2 with space group $B 1 \quad 12 / b$ by repeating the translation $\mathbf{a} / 4+\mathbf{b} / 2+\mathbf{c} / 2$. Repeating the translation $\mathbf{a} / 4-\mathbf{b} / 2+\mathbf{c} / 2$ leads to a twin orientation of MDO2. Structure refinement indicated a predominance of twinned MDO2 domains in the faulted crystal [35].

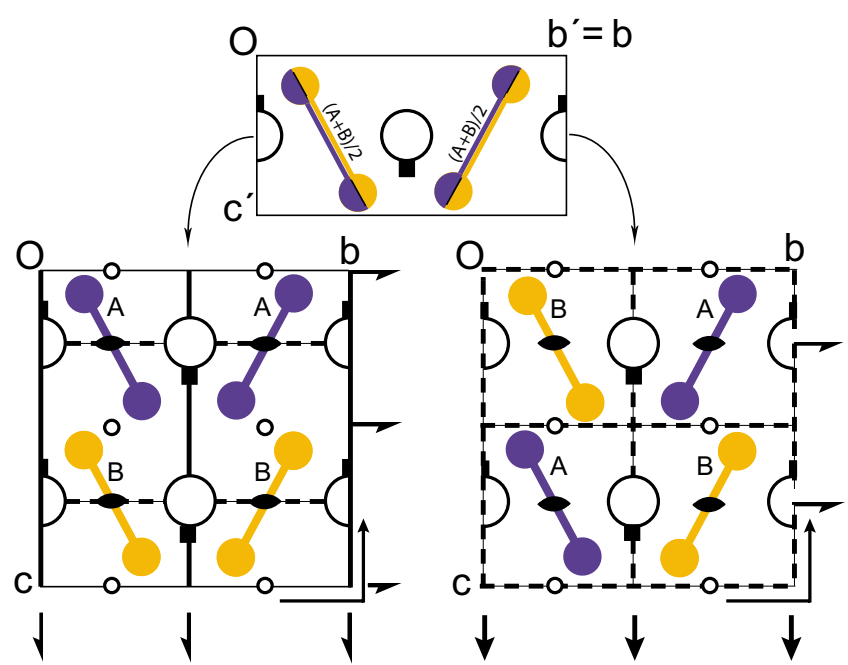

Fig. 7. Schematic drawing of two possible ways to disentangle the superposition structure obtained from the sharp reflections of the disordered phase of the (hexamethylenetetramine pimelic acid) adduct [36]. Top: A (blue) and B (yellow) represent the two superimposed orientations of the pimelic acid $\mathrm{C}_{7}$-chain relative to the plane of projection and with half occupation. The open circles with filles squares represent the hexamethylenetetramine molecules. Bottom: Two ways to disentangle a single layer with respective symmetries $p(2 / c) 2 / c 2 /$ $b$ (right) and $p(2 / c) 22_{1} / m 2_{1} / b$ (left). The $(c)$-glide plane transforms blue (yellow) into yellow (blue), the $c$ - and $b$-glide planes transform blue (yellow) into blue (yellow).

\subsection{The adduct of hexamethylenetetramine and pimelic acid}

This compound, $\left(\mathrm{C}_{6} \mathrm{H}_{12} \mathrm{~N}_{4}\right) \cdot\left[\mathrm{HOOC}\left(\mathrm{CH}_{2}\right)_{5} \mathrm{COOH}\right]$, runs through three phases on increasing temperature: $P 2_{1} / c$, $P 2_{1} / c 22_{1} / c 2 / n$ and a disordered orthorhombic phase [36]. Here we consider mainly the high-temperature orthorhombic phase and its relation to the other two phases. The sharp reflections in the high temperature phase have been indexed in a unit cell with $a^{\prime} \sim 9.4 \AA, b^{\prime} \sim 23.0 \AA, c^{\prime} \sim 7.3 \AA$. Diffuse rods run parallel to $\mathbf{a}^{\prime *}$ and require a unit mesh of $\mathbf{b}=\mathbf{b}^{\prime}, \mathbf{c}=2 \mathbf{c}^{\prime}$ for the layer structure. The superposition structure consists of two layers, symmetry $p(2 / m) 22_{1} / m$ $2 / b$, one as shown in Fig. 7 top and one displaced by $\left(\mathbf{a}^{\prime}+\mathbf{c}^{\prime}\right) / 2$. Two pimelic acid molecules $\mathrm{A}$ and $\mathrm{B}$, mirror related with respect to the $\left[\mathbf{b}^{\prime}, \mathbf{c}^{\prime}\right]$-plane, are superimposed on top of each other, but the carbon atoms of their $\mathrm{C}_{7^{-}}$ chains are resolved. In contrast to the previous example, there are two ways to disentangle these layers (Fig. 7, bottom). Chemically both possibilities are equally reasonable. The phases observed at intermediate and low temperature together with structural work on closely related compounds point to disentanglement at the bottom right of Fig. 7 with layer group $p(2 / c) 21 / c 2 / b$. Repeating the $2_{1}$ operation at $(x, 1 / 4,3 / 8)$ produces MDO1 with space group $P 2_{1} / c 2 / c 2 / n$. It is the intermediate temperature structure. Repeating the translation $(2 \mathbf{a}+\mathbf{c}) / 4$ produces $\mathrm{MDO} 2$ with space group $P 12_{1} / c$ 1. It is the low temperature structure. Repeating the translation $(2 \mathbf{a}-\mathbf{c}) / 4$ leads to a twin orientation of MDO2. Twinning was indeed observed experimentally. Note that the discussion in the original work uses an alternative monoclinic unit cell [36]. The intensity distribution along the diffuse rods has not been analysed. 

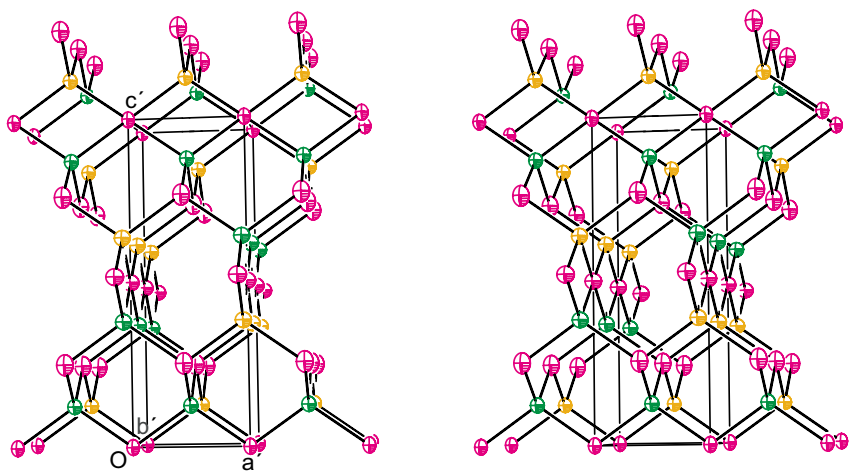

Fig. 8. Stereoscopic representation of the superposition structure of $\mathrm{HgI}_{2}$, space group $\mathrm{P}_{2} / m m c$. The $\mathrm{Hg}$-atoms are colored in yellow or green to facilitate following the arguments on the disentanglement of the superposition structure. In each double layer of edge sharing tetrahedra either the yellow or the green atoms must be removed (see text).

\subsection{Orange mercuric diiodide}

Schwarzenbach and Hostettler have investigated this compound in great detail $[33,34]$. Here we show that the data on the orange modification may be treated in a way that differs somewhat from the original analysis, but conforms entirely to the protocol described for $\mathbf{1}$. The scattering of orange $\mathrm{HgI}_{2}$ shows $4 / \mathrm{mmm}$ diffraction symmetry with diffuse rods parallel to $\mathbf{c}^{*}$. The sharp reflections define a lattice with $a^{\prime}=b^{\prime} \sim 4.4 \AA, c^{\prime} \sim 12.3 \AA$. The superposition structure, space group $P 4_{2} / \mathrm{mmc}$, determined from the Patterson function with SHELXS [37] is shown in Fig. 8. It is based on a cubic closest packed arrangement of iodine atoms. Double layers of edge sharing tetrahedra are arranged parallel to $\mathbf{a}$ and $\mathbf{b}$ and filled with half- $\mathrm{Hg}$ atoms. Disentanglement is guided by the requirements that the positions of the diffuse rods require a unit mesh $a=b=2 a^{\prime}$; the layer structure should give rise to only one kind of layer pair; $\mathrm{Hg}$ is expected to show tetrahedral coordination and $\mathrm{Hg}-\mathrm{I}-\mathrm{Hg}$ angles are tetrahedral [38]. There are two ways to define layers perpendicular to $\mathbf{c}^{\prime}$ in the superposition structure, either in terms of the slice bordered by the shared edges of the tetrahedra $\left(0 \leq z^{\prime} \leq 1 / 2\right)$, or in terms of the slice bordered by the corner-linking I atoms $\left(1 / 4 \leq z^{\prime} \leq 3 / 4\right)$. Disentangling the first possibility is discussed in detail in $[33,34]$. It leads to layers of cornersharing supertetrahedra and two kinds of layer pairs. The alternative slice is disentangled by removing the rows of yellow half-Hg atoms at $z=3 / 8$ and $5 / 8$. This eliminates edge sharing and leads to a slice with translations $\mathbf{a}=2 \mathbf{a}^{\prime}$, $\mathbf{b}=\mathbf{b}^{\prime}$, layer group pmm $(a)$. At $z=7 / 8$ and $9 / 8(=1 / 8)$ either the yellow or the green rows are removed. In both cases a slice with translation $\mathbf{a}=\mathbf{a}^{\prime}, \mathbf{b}=2 \mathbf{b}^{\prime}$ and layer group $\operatorname{pmm}(b)$ results. Adjacent slices are related by fourfold screw axes passing through one or the other of the two different iodine atoms $\left(z^{\prime}=1 / 4,3 / 4\right.$ or $\left.z^{\prime}=1 / 2\right)$. One of these generates MDO1 $\left(I_{1} /\right.$ amd $)$, the other one generates MDO2 $\left(\mathrm{P}_{2} / n m c\right)$ [34]. With this description all pairs of layers are geometrically equivalent, whereas there are two different kinds of triplets.

The structure of $\mathrm{KInS}_{2}$ and related compounds of the $\mathrm{TlGaSe}_{2}$-type has also been viewed as built from layers of corner-sharing supertetrahedra $\left(\mathrm{M}_{4} \mathrm{X}_{10}\right)$ [7, 39]. Although the relationship between subsequent layers of supertetrahedra differs from that in $\mathrm{HgI}_{2}$ due to the presence of the $\mathrm{K}$ ions, there are again two different kinds of interlayer contacts. However, in close analogy to orange $\mathrm{HgI}_{2}$, alternative layers may be chosen consisting of two parallel rows of simple tetrahedra related by a glide operation perpendicular to the rows. This choice, again with layer group $\operatorname{pmm}(a)(\operatorname{pmm}(b))$, ensures that all interlayer-contacts are geometrically equivalent with differences occurring only between next-nearest layers.

\section{$3.4 p$-(Trimethylammonio)benzenesulfonate}

Finally, we draw attention to three polymorphs built from the zwitterion ${ }^{-} \mathrm{O}_{3} \mathrm{~S}-\mathrm{C}_{6} \mathrm{H}_{4}-\mathrm{N}\left(\mathrm{CH}_{3}\right)_{3}{ }^{+}$[14]. All structures consist of molecular layers with the molecular dipoles perpendicular to the layers, alternating in direction, and arranged in a chessboard-like pattern. The low temperature polymorph is ordered; the intermediate and high temperature polymorphs show diffuse streaks corresponding to different degrees of stacking disorder. A closer inspection of the structures suggests that the best choice for analysing the disorder is not to use a single type of layer, but rather to consider two types of layers: ionic ones built from the entities $-\mathrm{N}\left(\mathrm{CH}_{3}\right)_{3}{ }^{+} \ldots{ }^{-} \mathrm{O}_{3} \mathrm{~S}-$ with layer symmetry $\operatorname{pbm}(a)$, and hydrophobic ones built from the fragments $-\mathrm{C}_{6} \mathrm{H}_{4}-$ with the lower layer symmetry $p 11(2)$. Apolar layers may be inserted between polar ones in four different, but geometrically equivalent ways. The experimental observations for the intermediate temperature phase imply that the orientation of the $-\mathrm{N}\left(\mathrm{CH}_{3}\right)_{3}{ }^{+} \ldots{ }^{-} \mathrm{O}_{3} \mathrm{~S}-$ fragments in successive polar layers does not change, whereas the relative orientation of the $-\mathrm{C}_{6} \mathrm{H}_{4}-$ fragments in successive apolar layers may differ. The apparent arduousness of the analysis engendered by having to consider a polar and an apolar layer is entirely compensated in realizing that the high temperature tetragonal phase may be explained by admitting different orientations not only for the molecular fragments in the apolar layers, but also for those in the polar ones, without, however, affecting the nearest-neighbour relationship between polar and apolar layers! A more recent study reports somewhat discrepant results [40]. While diffuse streaks parallel to the direction of the zwitterions have revealed stacking disorder in the first study [14], these streaks are not explicitly mentioned in the second one. It cannot be excluded that the crystal structures of the corresponding samples produced at different times, in different laboratories from different solvents are indeed somewhat different, as was clearly observed for 1 (Section 1).

\subsection{A few observations on the examples discussed above}

The first observation concerns bis(malondiamidine)-nickel(II) dichloride (Sect. 3.1) and the adduct of hexamethylenetetramine and pimelic acid (Sect. 3.2). Our discussions show the close analogy with the procedure applied to $\mathbf{1}$, and provide a reminder that disentanglement of the superposition structure is not always completely straightforward, and may require either a quantitative analysis of the 
diffuse scattering or independent structural information. Ways to include such information qualitatively and (semi)quantitatively have been discussed [3, 5, 41-43].

The second observation concerns the $\mathrm{HgI}_{2^{-}}$and $\mathrm{TlGaSe}_{2}$-type structures (Section 3.3) as well as the zwitterion example (Section 3.4). They clearly bring home the point that a choice of layers, which appears to be chemically significant, e.g. layers of supertetrahedra or of zwitterionic molecules, may not provide the most appropriate basis for a consistent analysis of stacking disorder. A choice with all nearest-neighbour relationships equivalent is to be preferred because it implies that the interactions between nearest neighbours are always the same, and that differences in stacking are related to more distant interactions. More formal, geometric criteria for choosing the layers have been described by Grell [44].

The third observation concerns the stacking disorder of $\mathbf{1}$ in relation to close packing of spheres (the literature on the latter topic is too vast to be referenced exhaustively here [31, 45-47]): if only the even (odd) layers of $\mathbf{1}$ are considered, the corresponding molecules can assume three positions analogous to the $A, B, C$ positions in close packed arrangements. Whereas in sphere packings, consecutive layers cannot have the same letter, stackings of the type $\cdots A A \cdots$ are possible for $\mathbf{1}$ because of the interspersed layers of (locally) inversion related molecules (eclipsed stacking, Sect. 2.2.2). We note that although the phenomenon of stacking disorder was originally observed in metallic and inorganic systems, it is also found for (nearly) spherical molecules, e.g. in the crystal structures of $\mathrm{C}_{70}$ [13], and even for a virus [24].

\section{Discussion}

\subsection{Crystal packing of tris(bicyclo[2.1.1]hexeno)benzene}

A structural chemist may want to know the average size of the three different MDO stacks possible for $\mathbf{1}$ and their relative contributions to the volume of the specimen investigated. The probabilities $P_{e}, P_{b}$ to find bent and eclipsed stacks anywhere in the structure follow from the relationships relating these probabilities to the probabilities of adding new layers, $P_{e}=e_{2} P_{e}+e_{1} P_{b}, P_{b}=\left(1-e_{e}\right) P_{e}+$ $\left(1-e_{1}\right) P_{b}, \quad P_{e}+P_{b}=1$. They are $P_{b}=\left(1-e_{2}\right) /$ $\left(1+e_{1}-e_{2}\right)$ and $P_{e}=e_{1} /\left(1+e_{1}-e_{2}\right)$. The probability $E_{n}$ to find a stack of $n$ eclipsed layers $(e)$ bounded on either side by bent arrangements $(b)$ equals the probability to find a bent arrangement in the first place times the nucleation probability for an eclipsed arrangement times the growth probabilities for an eclipsed arrangement times the probability to discontinue the eclipsed stack:

$$
E_{n}=\frac{1-e_{2}}{1-e_{2}+e_{1}} \cdot e_{1} \cdot\left(e_{2}\right)^{n-1} \cdot\left(1-e_{2}\right) .
$$

The average length of an MDO1 stack becomes

$$
\left\langle n_{e}\right\rangle=\frac{\sum n \cdot E_{n}}{\sum E_{n}}=\frac{1}{1-e_{2}} .
$$

In the present case with $e_{1}$ as small as $\sim 0.08$ only very few eclipsed stacks are formed and because $e_{2} \sim 0.56$, they are of limited length, mostly (beeb) or (beeeb), corresponding to 2-3 MDO1 unit cells.

The probabilities for finding cis and trans quadruplets are derived by analogous reasoning. The average stack lengths become $\left\langle n_{c}\right\rangle=(1-c)^{-1}$ and $\left\langle n_{t}\right\rangle=(1-t)^{-1}$. With $c \sim t \sim 0.46$ the average length of MDO2 and MDO3 are $\sim 2$. This implies that on average five layers stack into a mini-domain corresponding to less than a full unit cell for MDO2 and to 2.5 unit cells for MDO3. However the average length of a disordered cis-trans domain is much larger: $\left\langle n_{c, t}\right\rangle=(1-c-t)^{-1}$, i.e. about 12-13 layers.

From the stacking probabilities it follows that the eclipsed arrangement is disfavoured relative to the bent one and that continuing the bent stacking in a cis or a trans fashion is equally probable. The free energy differences between different stacking patterns are thus very small, less than a $\mathrm{kcal} / \mathrm{mol}$ if the crystallization process took place at room temperature. One might nevertheless ask whether the distinction between bent and eclipsed stacking could have a structural basis. Note from Fig. 5 that the ratio between small maxima at even $L$ and large ones at odd $L$ is well reproduced, whereas the observed large maxima do not always compare too well with the calculated ones. This may have two reasons. First, the layer form factor $F$ taken from the perfectly planar $p(\overline{6}) 2 m$ layer determined from a hexagonal disordered crystal in [26] may not be quite adequate. Indeed, in the nearly unfaulted monoclinic MDO3 polymorph also described in [26] the molecules of $\mathbf{1}$, which show crystallographic twofold symmetry (space group C2/ $m$ ), are slightly rotated about their twofold axes out of the $p(\overline{6}) 2 m$ plane. In the MDO2 structure $\left(P 6_{1} 22\right)$ the molecules also show twofold site symmetry, i.e. they are also free to rotate. In contrast the site symmetry of $\mathbf{1}$ in MDO1 is $m m 2$, i.e. the orientation of the molecule relative to the layer is fixed. Taken together these observations suggest that the molecules in the real crystal generally make use of the tilting degree of freedom, but are prevented from doing so, if they try to stack in an eclipsed fashion. The energetic cost involved in this restriction keeps $e_{1}$ small, but once the eclipsed stacking has been initiated and the layer planarized, the continuation in eclipsed stacking is less problematic, $e_{2}>e_{1}$. Second, if the real structure exhibits slight orientational disorder, the adp's in the c-direction will have two main contributions, one representing thermal motion and a second one reflecting static disorder in an averaged fashion. Simulating orientational disorder by adp's in an averaged way also hampers agreement between observed and calculated intensity profiles.

\subsection{Outlook}

In the work presented here a systematic protocol for determining structures with 1D stacking disorder has been summarized and applied to the analysis of diffuse streaks in the diffraction pattern of $\mathbf{1}$. The protocol has been compiled from several studies done over the last several decades, mostly on individual compounds or on classes of chemically closely related compounds. The discussion has also addressed - implicitly or explicitly - several limita- 
tions of the present approach for determining crystal structures with stacking disorder. Some are purely technical, e.g. the complicated algebra required to formulate a closed form of the interference function $S(L)$ based on sometimes quite complex Markov chains [33]. Others derive from a lack of appropriate software tools with sufficient flexibility to determine the probabilities and structural parameters necessary to satisfactorily model diffuse scattering. The use of numerical techniques such as genetic algorithms might be one way to alleviate some of these difficulties and to develop somewhat user-friendlier tools for analysing crystal structures with stacking disorder [48].

\section{Appendix}

We present here a classical derivation of the diffuse intensities on the rods $-h+k=3 n$ for the disordered crystals of 1 , without making use of advanced mathematics and adaptable to other layer structures (see also [33, 34]). First, the diffuse intensity function is written in terms of the relative orientations of all possible pairs of layers in the crystal and their probabilities of occurrence. There is a close relationship between this result and that obtained for closest sphere packing. Second, the probabilities for the occurrence of any pair of layers are expressed in terms of Markov chains involving near-neighbour stacking probabilities. Finally, these probabilities are translated into those used in the main text to obtain an explicit form of the interference function, Eq. (2).

We denote layers with the molecules oriented as shown in Fig. 3 by upper-case letters $X=A, B$ or $C$. Layers inverted through a centre of symmetry are denoted by lower-case letters $x=a, b$ or $c$. In an A-layer, the molecules are centred on the origin of the two-dimensional unit cell [a, b] (Fig. 4a). In $B$ - and $C$-layers they are displaced by the vectors $\mathbf{d}_{B}=[2 / 3,1 / 3]$ and $\mathbf{d}_{C}=[1 / 3,2 / 3]$, respectively, while $a, b$ and $c$ are displaced by $\mathbf{d}_{a}=[1 / 3,0], \mathbf{d}_{b}=[0$, $1 / 3]$ and $\mathbf{d}_{c}=[2 / 3,2 / 3]$, respectively. Any ordered or disordered structure is characterized by an alternating sequence of letters $X x X^{\prime} x^{\prime} \ldots$ The intensity $I\left(L_{1}\right)$ along a lattice row is given by [49]:

$$
\begin{aligned}
& I\left(L_{1}\right)=\sum_{m=-2 M+1}^{2 M-1}(2 M-|m|) J_{m} \exp \left(-2 \pi i L_{1} m\right), \\
& J_{m}=\sum_{r} \sum_{s} p_{r} P_{r s}^{(m)} F_{r} F_{s}^{*} .
\end{aligned}
$$

The coordinate $L_{1}$ refers to the spacing of single layers, $c^{\prime}=4.6 \AA$. The crystal comprises $2 M$ layers, $M$ of each type $X$ and $x$, and Eq. (3) accounts for $4 M^{2}$ distances between layers; $p_{r}$ is the a-priori probability of finding in the stack a layer of type $r ; P_{r s}^{(m)}$ is the a-posteriori probability of finding a layer of type $s, m$ layers from one of type $r$. Supposing that all layers occur with equal probability and are on average equivalent, $p_{r}=1 / 6$ for all $r$. Both partial stacks $X$ and $x$ show flocks of parallel threefold axes perpendicular to the planes, but in different positions. The stack of odd (even) layers may thus be inserted into the stack of even (odd) layers in three equivalent ways. Due to this symmetry, which agrees with the diffraction symmetry $6 / m m m, P_{X x}^{(m)}=P_{x X}^{(m)}=1 / 3$ for all $m$ odd. Similarly the mirror symmetry between the two non-eclipsed positions of next-nearest neighbours insures $P_{X Y}^{(m)}=P_{x y}^{(m)}$ $=\left(1-P_{X X}^{(m)}\right) / 2$ for $X \neq \mathrm{Y}, x \neq y$ and all $m$ even. For each even interlayer distance $m$ there remains one independent a-posteriori probability $P_{X X}^{(m)}=P_{m}$ to find two layers with the same position. $F_{r}$ is the structure factor of a layer of type $r$. Specifically, $F_{X}=F_{A} \exp \left(2 \pi \mathrm{ih} \cdot \mathbf{d}_{X}\right)$ and $F_{x}=F_{A}^{*}$ $\exp \left(2 \pi \mathrm{ih} \cdot \mathbf{d}_{x}\right), \mathbf{h}$ being the vector $(h, k)$ and $F^{*}$ the complex conjugate of $F$. Developing explicitly all terms of Eq. (4) leads to $J_{m}=\left|F_{A}\right|^{2}\left(3 P_{m}-1\right) / 2$ for $m$ even, and 0 for $m$ odd. $P_{m}$ converges towards $1 / 3$ for large $m$ except for ordered structures with translational symmetry. We therefore extend the sum in Eq. (3) to infinity. We also double the $c$-axis, $c=2 c^{\prime}, L_{2}=2 L_{1}$, which now comprises two layers and obtain for Eq. (1)

$$
\begin{aligned}
& I\left(L_{2}\right)=2 M\left|F_{A}\right|^{2}\left\{1+2 \sum_{1}^{\infty} Q_{m} \cos 2 \pi m L_{2}\right\}, \\
& Q_{m}=\left(3 P_{m}-1\right) / 2 .
\end{aligned}
$$

This equation is identical to the one obtained for a closest sphere packing. Although the allowed relative positions of layers $X$ and $x$ are completely random, interference between them does not contribute to the diffuse intensity, but does contribute to the family reflections.

We now express the $P_{m}$ in terms of near-neighbour probabilities with a depth of interaction of two layers in the formalism of densest sphere packings. The derivation follows closely [32] with the complication that the probability for nearest neighbours to occupy the same position is $P_{1} \neq 0$. The basic probabilities are analogous to the ones described in Section 2.2.3: $\alpha_{1}$ for $B A A$ (create eclipsed stack), $\beta$ for $B A B$ ("hexagonal" stack), $\gamma$ for $B A C$ ("cubic" stack), $\alpha_{2}$ for $A A A$ (continue eclipsed stack); $\alpha_{1}+\beta+\gamma=1,0 \leq \alpha_{2} \leq 1$.

Layer 0 is now assumed to have position $A$. We define a vector $\mathbf{v}_{m}=\left(a_{m}, a_{m}^{\prime}, a^{\prime \prime}{ }_{m}, b_{m}, b_{m}^{\prime}, b_{m}^{\prime \prime}, c_{m}, c_{m}^{\prime}, c^{\prime \prime}{ }_{m}\right)$ whose terms are the probabilities that layers $m-1$ and $m$ occupy positions $A A, C A, B A, B B, A B, C B, C C, B C, A C$, respectively. The corresponding Markov relationship is

$$
\begin{aligned}
& \mathbf{v}_{m}=\mathbf{v}_{m-1} \mathbf{O}=\mathbf{v}_{1} \mathbf{O}^{m-1}, \\
& \mathbf{v}_{1}=\left(P_{1}, 0,0,0,\left(1-P_{1}\right) / 2,0,0,0,\left(1-P_{1}\right) / 2\right),
\end{aligned}
$$

where the elements of the 9 times 9 Markov matrix $\mathbf{O}$ are either $\alpha_{1}, \alpha_{2},\left(1-\alpha_{2}\right), \beta, \gamma$ or 0 . The following relations may be demonstrated from the recursive relationships between $\mathbf{v}_{m}$ and $\mathbf{v}_{m-1}$ :

$$
\begin{aligned}
& a_{m}+a_{m}^{\prime}+a_{m}^{\prime \prime}=P_{m}, \\
& b_{m}+b_{m}^{\prime}+b_{m}^{\prime \prime}=\left(1-P_{m}\right) / 2=c_{m}+c_{m}^{\prime}+c_{m}^{\prime \prime}, \\
& a_{m}+b_{m}+c_{m}=P_{1}, \\
& a_{m}^{\prime}=a_{m}^{\prime \prime}, \quad b_{m}=c_{m}, \quad b_{m}^{\prime}=c_{m}^{\prime \prime}, \quad b_{m}^{\prime \prime}=c_{m}^{\prime}, \\
& a_{m+1}+b_{m+1}^{\prime}+c_{m+1}^{\prime \prime}=P_{m}, \\
& a_{m+1}^{\prime}+b_{m+1}^{\prime \prime}+c_{m+1}=\left(1-P_{m}\right) / 2 \\
& =a_{m+1}^{\prime \prime}+b_{m+1}+c_{m+1}^{\prime}
\end{aligned}
$$


and allow us to arrive at the result

$$
\begin{aligned}
& Q_{m}=D Q_{m-1}+E Q_{m-2}+G Q_{m-3} \\
& D=\alpha_{2}-\gamma, \\
& E=\left[\left(\alpha_{2}-\alpha_{1}\right)-\left(\alpha_{2}-2 \beta-2 \gamma\right)(\beta-\gamma)\right] / 2, \\
& G=\left(\alpha_{1}-\alpha_{2}\right)(\beta-\gamma) \\
& P_{0}=1, \quad P_{1}=\alpha_{1} /\left(1+\alpha_{1}-\alpha_{2}\right), \\
& P_{2}=\left(\alpha_{2}-\beta\right) P_{1}+\beta,
\end{aligned}
$$

where Eq. (6c) is derived as described at the beginning of Section 4.1. For $\alpha_{1}=\alpha_{2}=0$, we obtain the well-known formula for the closest sphere packing [32]. Equation (6a) can be written in Markov form:

$$
\begin{aligned}
& \left(\begin{array}{c}
Q_{m} \\
Q_{m-1} \\
Q_{m-2}
\end{array}\right)=\mathbf{M}\left(\begin{array}{l}
Q_{m-1} \\
Q_{m-2} \\
Q_{m-3}
\end{array}\right)=\mathbf{M}^{m-2}\left(\begin{array}{c}
Q_{2} \\
Q_{1} \\
Q_{0}
\end{array}\right), \\
& \mathbf{M}=\left(\begin{array}{lll}
D & E & G \\
1 & 0 & 0 \\
0 & 1 & 0
\end{array}\right) .
\end{aligned}
$$

The summation of Eq. (5) is then carried out by strictly the same method as described in [34], and the coefficients of Eq. (1) are evaluated in terms of $\alpha_{1}, \alpha_{2}, \beta, \gamma$.

Finally, the probabilities $\alpha_{1}, \alpha_{2}, \beta, \gamma$ are expressed as functions of the probabilities $e_{1}, e_{2}, t$ and $c$. The sequence of type $A A A$ defining $\alpha_{2}$ is obtained from the sequences of type $A a A a A$ realized with probability $e_{2}{ }^{2}, A a A b A$ and $A a A c A$, each of which is realized with probability $\left(1-e_{2}\right)$ $e_{1} / 2$. Similarly, $B A A$ defining $\alpha_{1}$ results from $B a A a A$ $\left(e_{1} e_{2}\right), B a A b A$ (ce $\left.e_{1}\right)$ and $B a A c A\left(t e_{1}\right)$. Analogous arguments for $\beta$ and $\gamma$ lead to the desired relations:

$$
\begin{aligned}
& \alpha_{1}=e_{1}\left(1-e_{1}+e_{2}\right), \quad \beta=e_{1}\left(1-e_{2}\right) / 2+2 t c, \\
& \gamma=e_{1}\left(1-e_{2}\right) / 2+t^{2}+c^{2}, \quad \alpha_{2}=e_{1}-e_{1} e_{2}+e_{2}{ }^{2} .
\end{aligned}
$$

This restricts the probabilities $\beta$ and $\gamma$ to $0 \leq \beta \leq 1 / 2$, $1 / 2 \leq \gamma \leq 1$. In terms of closest sphere packings, possible sequences of layers thus range from completely disordered to ordered "cubic" $A B C$, but not to ordered "hexagonal" $A B A$. Indeed, both $\mathrm{MDO} 2$ and $\mathrm{MDO} 3$ show "cubic" sequences. A "hexagonal" sequence arises in the ordered structure obtained with alternating trans and cis stacks, but this is not an MDO. The final result for Eq. (2) is

$$
\begin{aligned}
S\left(L_{2}\right)= & \frac{\left(T+U \cos 2 \pi L_{2}+V \cos 4 \pi L_{2}\right)}{\left(W+X \cos 2 \pi L_{2}+Y \cos 4 \pi L_{2}+Z \cos 6 \pi L_{2}\right)} \\
T=1 & +D^{2}+E^{2}-G^{2}-2 Q_{1} D+2 Q_{1} D E-2 Q_{2} E \\
U= & -2 D+2 D E+2 Q_{1}+2 Q_{1} D^{2}-2 Q_{1} E+2 Q_{1} D G \\
& -2 Q_{2} D-2 Q_{2} G \\
V= & -2 E-2 Q_{1} D-2 Q_{1} G+2 Q_{2} \\
W= & 1+D^{2}+E^{2}+G^{2} \\
X= & 2(-D+D E+E G) \\
Y= & 2(-E+D G) \\
Z= & -2 G
\end{aligned}
$$

$$
\begin{aligned}
D= & e_{1}\left(1-e_{2}\right) / 2+e_{2}^{2}-\left(t^{2}+c^{2}\right) \\
E= & {\left[\left(e_{1}-e_{2}\right)^{2}+\left(e_{2}^{2}-2 e_{1}^{2}+e_{1} e_{2}+3 e_{1}-2\right)\right.} \\
& \left.\times(t-c)^{2}\right] / 2 \\
G= & \left(e_{1}-e_{2}\right)^{2}(t-c)^{2} \\
Q_{1}= & \left(2 e_{1}+e_{2}-1\right) /\left[2\left(1+e_{1}-e_{2}\right)\right] \\
Q_{2}= & \left\{\left(1-e_{2}\right)\left[3 e_{1}\left(e_{1}-e_{2}\right)+6 t c-1\right]\right. \\
& \left.+\left(e_{1} / 2\right)\left(3 e_{2}^{2}+1\right)\right\} /\left[2\left(1+e_{1}-e_{2}\right) .\right.
\end{aligned}
$$

Eq. (2) represents the intensity not as a function of $L_{2}$, but instead as a function of $L=L_{4}=2 L_{2}$ which corresponds to $c=18.4 \AA$ comprising 4 single layer spacings. The coefficients $T$ to $Q_{2}$ remain of course unchanged. These tedious (but well-checked) equations may also be used with the coefficients of Eq. (6) and give then an explicit formula for disordered sphere packings which to our knowledge has not been published before.

It may be of interest that the same diffuse intensity is obtained for some values $t=p, c=q$ and the interchanged values $t=q, c=p$. Indeed, the diffraction by an ordered MDO2 crystal (neglecting anomalous scattering) is identical to the diffraction by an equi-domain 6-fold twinned crystal of MDO3. Although such crystals have not been found yet, this shows that the interpretation of diffuse scattering and of twinned crystals may be ambiguous (see also [34, 38]). Since our diffraction data give $t=c$, there is no such ambiguity in our interpretation.

Acknowledgments. We thank Prof. K. Komatsu for providing crystals of $\mathbf{1}$ and Prof. H. D. Flack for helpful discussions and insightful suggestions. Support for this research from the Swiss National Science Foundation is gratefully acknowledged. H.B. thanks the Danish Research Councils for additional support of this work and for a Steno Research Assistant Professorship funded by the Danish Natural Science Research Council and the Danish Technical Research Council under contract (21-03-0433).

Supplementary material available from the online edition and from the website of the authors http://www.krist.unibe.ch/publication.html

\section{References}

[1] Moss, D. S.; Harris, G. W.; Wostrack, A.; Sansom, C.: Diffuse X-ray scattering from molecular crystals. Crystallog. Rev. 9 (2003) 229-277.

[2] Frey, F.: Diffuse scattering from periodic and aperiodic crystals. Z. Kristallogr. 212 (1997) 257-282.

[3] Welberry, T. R.; Butler, B. D.: Diffuse X-ray scattering from disordered crystals. Chem. Rev. 95 (1995) 2369-2403.

[4] Cowley, J. M.: Diffraction physics. Elsevier Science B. V. 1995.

[5] Jagodzinski, H.; Frey, F.: Disorder diffuse scattering of X-rays and neutrons. In: International Tables of Crystallography Vol. B Reciprocal Space (Ed. U. Shmueli), pp. 392-432. The International Union of Crystallography and Kluwer Academic Publishers 1993.

[6] Guinier, A.: X-ray diffraction in crystals, imperfect crystals, and amorphous bodies. Freeman, San Francisco and London, 1963.

[7] Kienle, L.; Duppel, V.; Simon, A.; Schlosser, M.; Jarchow, O.: Real structure of $\mathrm{KInS}_{2}$ polytypes. J. Solid State Chem. 177 (2004) 6-16.

[8] Kim, J.; Hughbanks, T.: Synthesis and structures of ternary chalcogenides of aluminium and gallium with stacking faults: $\mathrm{KMQ}_{2}$ $(\mathrm{M}=\mathrm{Al}, \mathrm{Ga} ; \mathrm{Q}=\mathrm{Se}, \mathrm{Te})$. J. Solid State Chem. 149 (2000) $242-251$.

[9] Trigunayat, G. C.: A layer structure with unusual symmetry. Nature 212 (1966) 808-809. 
[10] Jagodzinski, H.: Eindimensionale Fehlordnung in Kristallen und ihr Einfluss auf die Röntgeninterferenzen. III. Vergleich der Berechnungen mit experimentellen Ergebnissen. Acta Crystallogr. 2 (1949) 298-304.

[11] Ferraris, G.; Makovicky, E.; Merlino, S.: Crystallography of Modular Materials, Oxford University Press, 2004.

[12] Bonin, M.; Welberry, T. R.; Hostettler, M.; Gardon, M.; Birkedal, H.; Chapuis, G.; Möckli, P.; Ogle, C. A.; Schenk, K.: Urotropin azelate: a rather unwilling co-crystal. Acta Crystallogr. B59 (2003) 72-86.

[13] Blanc, E.; Bürgi, H.-B.; Restori, R.; Schwarzenbach, D.; Ochsenbein, P.: X-ray diffraction study of the stacking faults in hexagonal $C_{70}$ single crystals. Europhys. Lett. 33 (1996) 205-210.

[14] Sarma, J. A. R. P.; Dunitz, J. D.: Structure of three crystalline phases of $p$-(trimethylammonio)benzenesulfonate and their interconversions. Acta Crystallogr. B46 (1990) 784-794.

[15] Flack, H. D.: Interpretation of anomalous streaks in crystals of anthrone. Acta Crystallogr. A26 (1970) 678-680.

[16] Lonsdale, K.; Nave, E.; Stephens, J. F.: X-ray studies of a single crystal chemical reaction: photo-oxide of anthracene to (anthraquinone, anthrone). Phil. Trans. A261 (1966) 1-31.

[17] Halla, F.; Jagodzinski, H.; Ruston, W. R.: One-dimensional disorder in dodecahydrotriphenylene, $\mathrm{C}_{18} \mathrm{H}_{24}$. Acta Crystallogr. 6 (1953) 478-486.

[18] Kasper, J. S.; Lucht, C. M.; Harker, D.: The crystal structure of decaborane, $\mathrm{B}_{10} \mathrm{H}_{14}$. Acta Crystallogr. 3 (1950) 436-455.

[19] Harata, K.; Akiba, T.: Phase transition of triclinic hen egg-white lysozyme crystal associated with sodium binding. Acta Crystallogr. D60 (2004) 630-637.

[20] Mancini, E. J.; Grimes, J. M.; Malby, R.; Sutton, G. C.; Kainov, D. E.; Juuti, J. T.; Makeyev, E. V.; Tuma, R.; Bamford, D. H.; Stuart, D. I.: Order and disorder in crystals of hexameric NTPases from dsRNA bacteriophages. Acta Crystallogr. D59 (2003) 2337-2341.

[21] Wall, M. E.; Clarage, J. B.; Phillips, G. N.: Motions of calmodulin characterized using both Bragg and diffuse X-ray scattering. Structure 5 (1997) 1599-1612.

[22] Pickersgill, R. W.: One-dimensional disorder in spinach ribulose bisphosphate carboxylase crystals. Acta Crystallogr. A43 (1987) 502-506.

[23] Kolatkar, A. R.; Clarage, J. B.; Phillips, Jr., G. N.: Analysis of diffuse scattering from yeast initiator tRNA crystals. Acta Crystallogr. D50 (1994) 210-218.

[24] Valegård, K.; Fridborg, V. K.; Liljas, L.: Crystallization and preliminary X-ray diffraction studies of the bacteriophage $\mathrm{Q}^{\beta}$. Acta Crystallogr. D50 (1994) 105-109.

[25] Bürgi, H.-B.; Baldridge, K. K.; Hardcastle, K.; Frank, N. L.; Gantzel, P.; Siegel, J. S.; Ziller, J.: X-ray diffraction evidence for a cyclohexatriene motif in the molecular structure of tris(bicyclo[2.1.1]hexeno)benzene: bond alternation after the refutation of the Mills-Nixon theory. Angew. Chem. Int. Ed. 34 (1995) 1454-1456.

[26] Birkedal, H.; Bürgi, H.-B.; Komatsu, K.; Schwarzenbach, D.: Polymorphism and stacking disorder in tris(bicyclo[2.1.1] hexeno)benzene. J. Mol. Struct. 647 (2003) 233-242.

[27] Oxford Diffraction, Xcalibur System. CrysAlis Software Package. Version 1.169. Oxfordshire, UK, 2003.

[28] WaveMetrics Inc. IGOR Pro Software. Version 4.0. Oregon, USA, 2000.

[29] Dornberger-Schiff, K.: Geometrical properties of MDO polytypes and procedures for their derivation. I. General concept and applications to polytype families consisting of OD layers all of the same kind. Acta Crystallogr. A38 (1982) 483-491.
[30] Dornberger-Schiff, K.; Grell H.: Geometrical properties of MDO polytypes and procedures for their derivation. II. OD families containing OD layers of $M>1$ kinds and their MDO polytypes. Acta Crystallogr. A38 (1982) 491-498.

[31] Jagodzinski, H.: Eindimensionale Fehlordnung in Kristallen und ihr Einfluss auf die Röntgeninterferenzen. I. Berechnung des Fehlordnungsgrades aus den Röntgenintensitäten. Acta Crystallogr. 2 (1949) 201-207.

[32] Jagodzinski, H.: Eindimensionale Fehlordnung in Kristallen und ihr Einfluss auf die Röntgeninterferenzen. II. Berechnung der fehlgeordneten dichtesten Kugelpackungen mit Wechselwirkungen der Reichwerte 3. Acta Crystallogr. 2 (1949) 208-214.

[33] Schwarzenbach, D.: The crystal structure and one-dimensional disorder of the orange modification of $\mathrm{HgI}_{2}$. Z. Kristallogr. 128 (1969) 97-114.

[34] Hostettler, M.; Birkedal, H.; Schwarzenbach, D.: The structure of orange $\mathrm{HgI}_{2}$. I. Polytypic layer structure. Acta Crystallogr. B58 (2002) 903-913.

[35] Schwarzenbach, D.; Schmelczer, R.: Comparison of an OD and an ordered structure of nickel and copper malondiamidine complexes. Acta Crystallogr. B34 (1978) 1827-1833. (Note that $b_{o}$ in the discussion of Schwarzenbach and Schmelczer is twice as long as the one used here).

[36] Gardon, M.; Pinheiro, C.B.; Chapuis, G.: Structural phases of hexamethylenetetramine-pimelic acid (1/1), a unified description based on a stacking model. Acta Crystallogr. B59 (2003) 527-536.

[37] Sheldrick, G. M.: SHELXL97, University of Göttingen, Germany, 1997.

[38] Hostettler, M.; Schwarzenbach, D.: The structure of orange $\mathrm{HgI}_{2}$. II. Diamond type structure and twinning. Acta Crystallogr. B58 (2002) 914-920.

[39] Müller, D.; Hahn, H.: Zur Struktur des $\mathrm{TlGaSe}_{2}$. Z. anorg. allg. Chem. 438 (1978) 258-272.

[40] Even, J.; Bertault, M.; Toupet, L.; Girard, A.; Kusto, W. J.: High temperature phase transitions in the crystal of $p$-trimethylammoniumbenzenesulfonate zwitterion. Eur. Phys. J. B12 (1999) 479-491.

[41] Müller, U.: Ein einfacher Weg, Strukturinformation aus den diffusen Reflexen von Kristallen mit eindimensionaler Lagenfehlordnung zu erhalten. Acta Crystallogr. A35 (1979) 957-961.

[42] Müller, U.: Die Kristallstruktur von Tantalpentajodid und ihre Fehlordnung. Acta Crystallogr. B35 (1979) 2502-2509.

[43] Frey, F.; Boysen, H.: Low dimensional diffuse scattering. Phase Transitions 67 (1998) 245-276.

[44] Grell, H.: How to Choose OD Layers. Acta Crystallogr A40 (1984) 95-99.

[45] Berliner, R.; Werner, S. A.: Effect of stacking faults on diffraction: the structure of lithium metal. Phys. Rev. B34 (1986) 3586-3603.

[46] Gevers, R.: X-ray diffraction by close-packed crystals with growth stacking faults assuming an n-layer influence. Acta Crystallogr. 7 (1954) 492-494.

[47] Gevers, R.: The diffraction of X-rays by close-packed crystals containing both 'growth stacking faults' and 'deformation or transformation stacking faults'. Acta Crystallogr. 7 (1954) 337343.

[48] Weber, Th.; Bürgi, H.-B.: Determination and refinement of disordered crystal structures using evolutionary algorithms in combination with Monte Carlo methods. Acta Crystallogr. A58 (2002) 526-540.

[49] Jagodzinski, H.: Der Symmetrieeinfluss auf den allgemeinen Lösungsansatz eindimensionaler Fehlordnungsprobleme. Acta Crystallogr. 7 (1954) 17-25. 


\section{Deposited supplementary material - only accessible online}
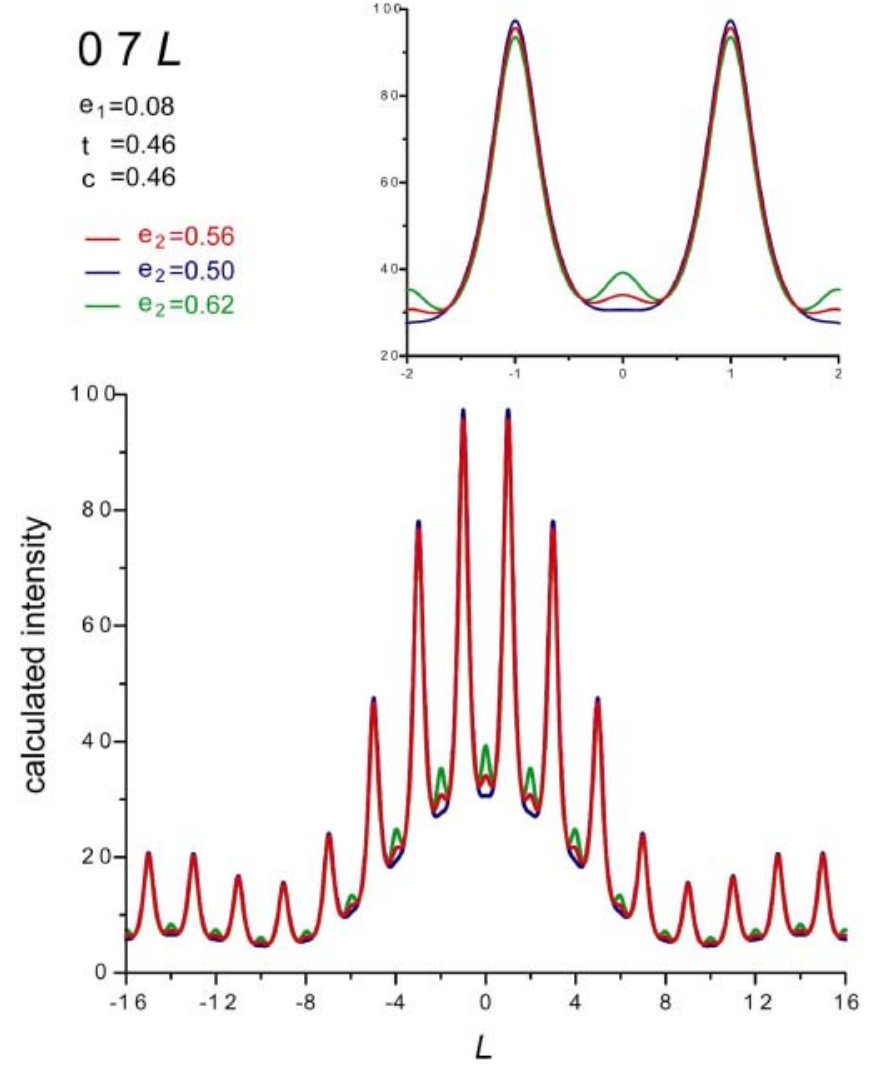

Fig. Sup. 1. Influence of small variations of the probabilities on the calculated profiles. Note that the probabilities $e_{1}, t$ and $c$ are constrained to fulfilled the relation $e_{1}+t+c=1$.
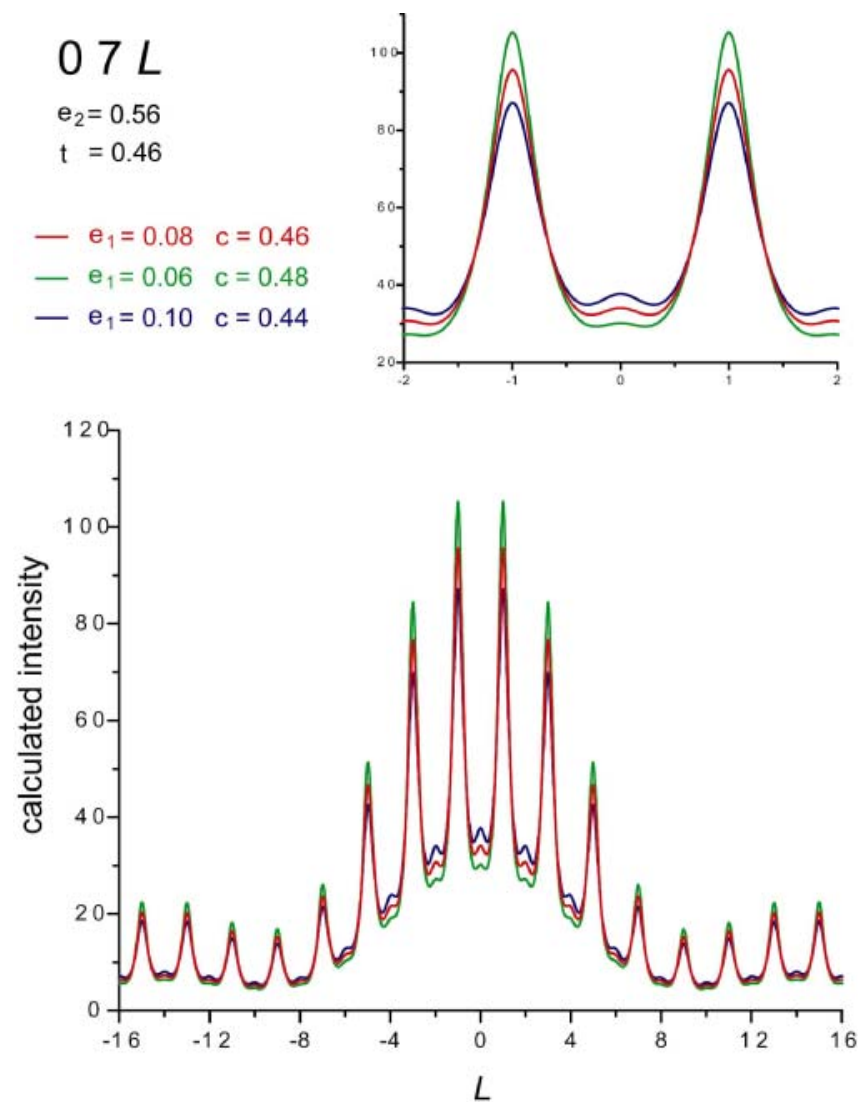

Fig. Sup. 2. Influence of small variations of the probabilities on the calculated profiles. Note that the probabilities $e_{1}, t$ and $c$ are constrained by the relation $e_{1}+t+c=1$. 


\section{$07 L$}

$\mathrm{e}_{2}=0.56$

$\mathrm{e}_{1}=0.08$

$-\mathrm{t}=0.46 \quad \mathrm{c}=0.46$

$-\mathrm{t}=0.36 \mathrm{c}=0.56$

$\mathrm{t}=0.56 \quad \mathrm{c}=0.36$

$-\mathrm{t}=0.26 \mathrm{c}=0.66$

$\mathrm{t}=0.66 \quad \mathrm{c}=0.26$

$-\mathrm{t}=0.16 \quad \mathrm{c}=0.76$

$\mathrm{t}=0.76 \quad \mathrm{c}=0.16$
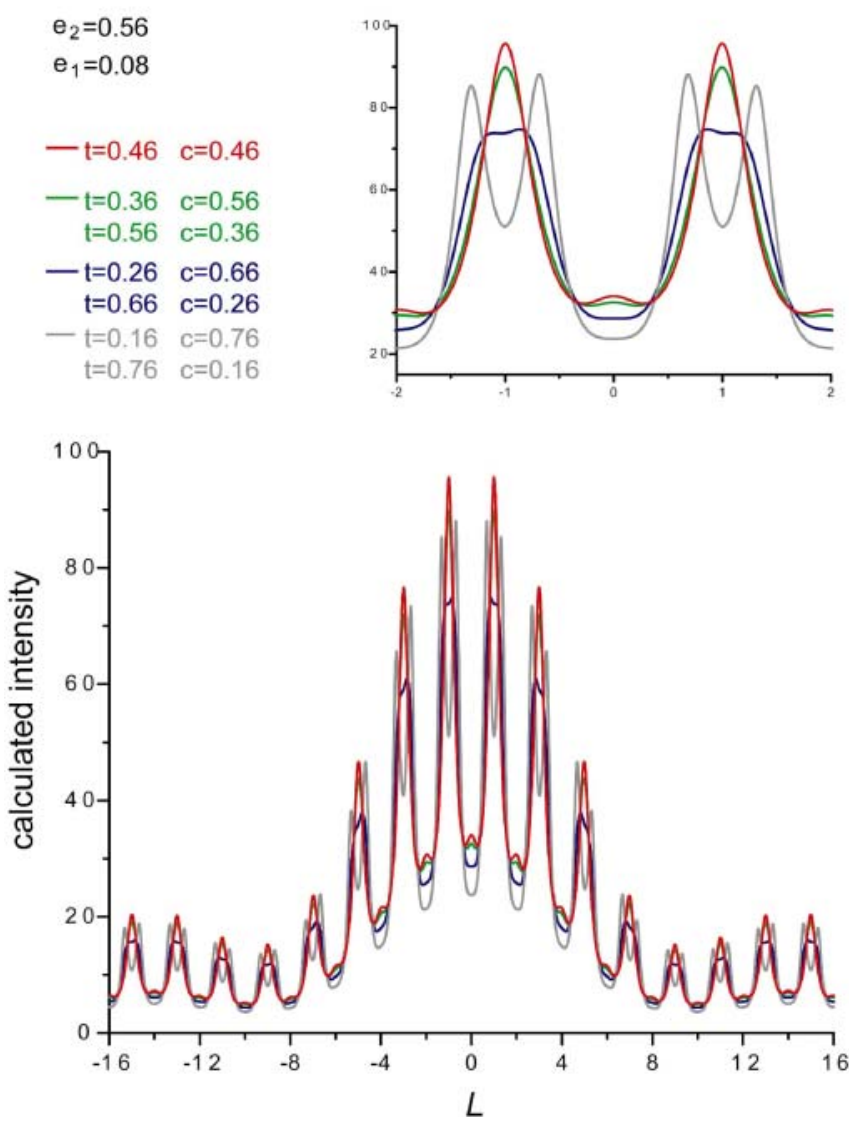

Fig. Sup. 3. Influence of small variations of the probabilities on the calculated profiles. Note that the probabilities $e_{1}, t$ and $c$ are constrained by the relation $e_{1}+t+c=1$. 

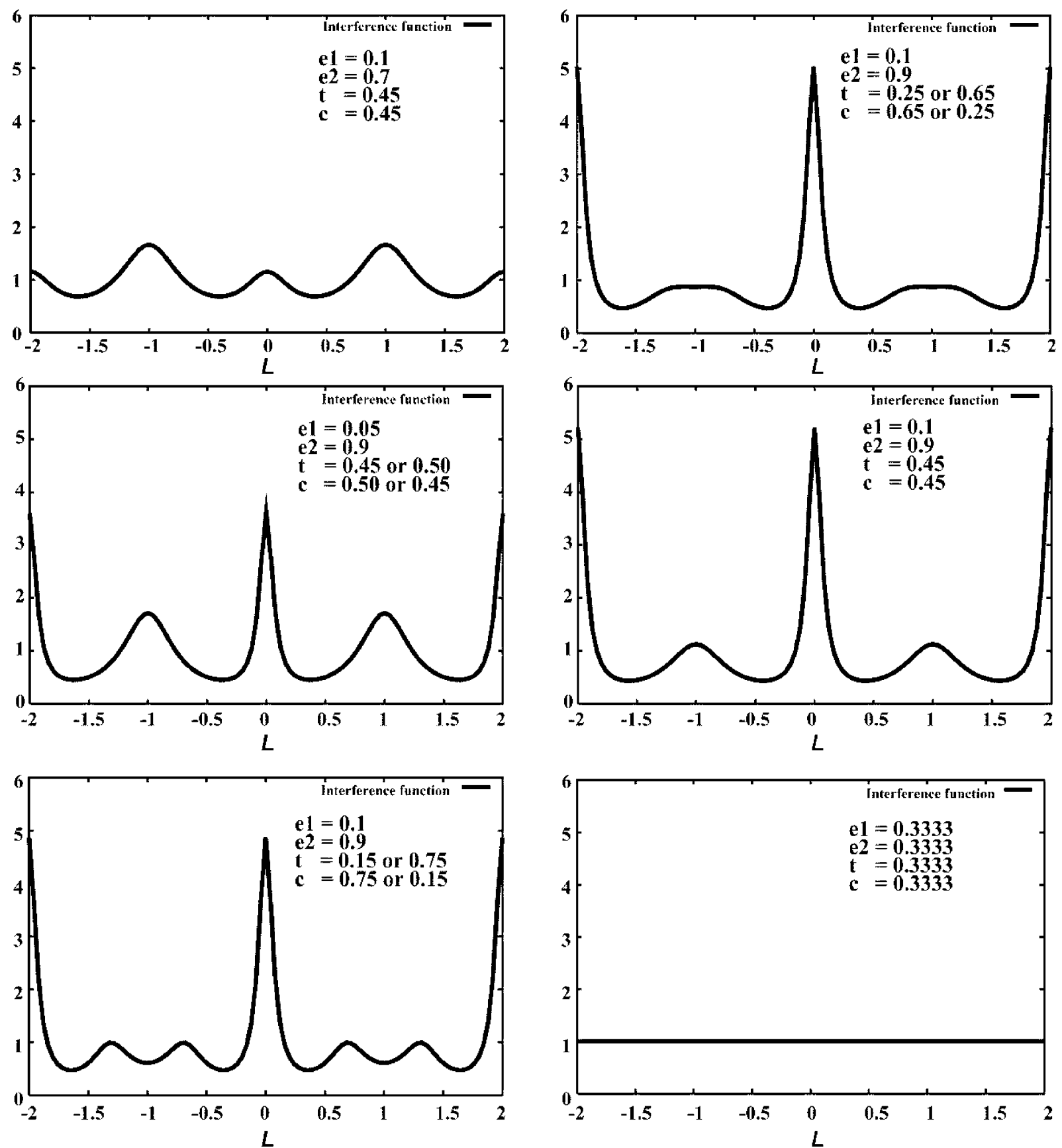

Fig. Sup. 4. The properties of the interference function for different sets of probabilties. Note that if all probabilities equal $1 / 3$, i.e. for a completely disordered case, $S(L)$ is flat implying that the diffuse intensity profiles would be determined by the layer from factor only. Note also that $S(L)$ is the same for $(t, c)$ and $(c, t)$, see the Appendix. 